\title{
CRMP-5 interacts with actin to regulate neurite outgrowth
}

\author{
XIAOBING GONG $^{1 *}$, MINGHUI TAN $^{2,3^{*}}$, YUAN GAO $^{1}, \mathrm{KEEN} \mathrm{CHEN}^{2,4}$ and GUOQING GUO ${ }^{2}$ \\ ${ }^{1}$ Department of Gastroenterology, The First Affiliated Hospital of Jinan University, Guangzhou, Guangdong 510630; \\ ${ }^{2}$ Department of Anatomy; ${ }^{3}$ Postdoctoral Stations of Integrated Traditional and Western Medicine, \\ Medical College of Jinan University, Guangzhou, Guangdong 510630; ${ }^{4}$ Department of Neurosurgery, \\ First Affiliated Hospital of Jinan University, Guangzhou, Guangdong 510630, P.R. China
}

Received January 19, 2015; Accepted October 29, 2015

DOI: $10.3892 / \mathrm{mmr} .2015 .4662$

\begin{abstract}
CRMP family proteins (CRMPs) are abundantly expressed in the developing nervous system mediating growth cone guidance, neuronal polarity and axon elongation. CRMP-5 has been indicated to serve a critical role in neurite outgrowth. However, the detailed mechanisms of how CRMP-5 regulates neurite outgrowth remain unclear. In the current study, co-immunoprecipitation was used to identify the fact that CRMP-5 interacted with the actin and tubulin cytoskeleton networks in the growth cones of developing hippocampal neurons. CRMP-5 exhibited increased affinity towards actin when compared with microtubules. Immunocy tochemistry was used to identify the fact that CRMP-5 colocalized with actin predominantly in the $\mathrm{C}$-domain and $\mathrm{T}$-zone in growth cones. In addition, genetic inhibition of CRMP-5 by siRNA suppressed the expression of actin, growth cone development and neurite outgrowth. Overexpression of CRMP-5 promoted the interaction with actin, growth cone development and hippocampal neurite outgrowth. Taken together, these data suggest that CRMP-5 is able to interact with the actin cytoskeleton network in the growth cone and affect growth cone development and neurite outgrowth via this interaction in developing hippocampal neurons.
\end{abstract}

\section{Introduction}

The neuronal system is complex, and synaptic contacts between polarized neurons are fundamental structures $(1,2)$. During

Correspondence to: Professor Guoqing Guo, Department of Anatomy, Medical College of Jinan University, 601 Huangpu Avenue West, Guangzhou, Guangdong 510630, P.R. China

E-mail: tgqguo@yahoo.com

*Contributed equally

Abbreviations: CRMP, collapsin response mediator protein; MTs, microtubules; DIV, days in vitro; NC, negative control; T-zone, the transition zone; $\mathrm{C}$-domain, the central domain

Key words: CRMP-5, actin, growth cone, hippocampal neuron, neurite growth the polarization process, neurons extend several neurites, the majority of which develop into dendrites and only one of which becomes an axon (3). Neurites are tipped with motile growth cones that receive extracellular guidance cues to reach their targets, thus forming neural circuits (4). Growth cones maintain the mobility predominantly due to the rich and flexible cytoskeleton, which contains microtubules and actin (5). Bundled microtubules are distributed predominantly in the central (C-) domain of the growth cone, while splayed or looped microtubules are predominantly in the peripheral (P-) domain and the transitional (T-) zone (6). Actin expresses predominantly in the P-domain and T-zone (7). When the growth cone is in a quiescent state, the polymerization and depolymerization of microtubules and actin exhibit a dynamic balance (8). If the balance is disrupted, the development of growth cone is affected.

Collapsin response mediator proteins (CRMPs), consisting of five cytosolic proteins (CRMP 1-5), are highly expressed in developing and adult nervous systems (9-11). CRMPs were originally identified as downstream of the Semaphorin-3A (Sema3A) signaling pathway (12). CRMP-5, as the first identified CRMP-associated protein, has the lowest homology with other CRMP proteins (13). CRMP-5 is highly expressed in post-mitotic neural precursors and the fasciculi of fibers in developing brains (14). Hotta et al (15) observed that CRMP-5 is able to regulate filopodia dynamics and growth cone development, negatively responding to Sema3A signaling. Yamashita et al (16) demonstrated that CRMP-5 regulated dendritic development and synaptic plasticity in the cerebellar Purkinje cells. However, conversely, CRMP-5 has been reported to inhibit neurite outgrowth (17). In addition, CRMP-5 antagonized the promoting effect of CRMP-2 on axonal and dendritic growth through a tubulin-based mechanism (17). Thus, the role of CRMP-5 in neurite outgrow th remains controversial. In addition, CRMP-5 is able to interact with other proteins during brain development, including tyrosine kinase Fes/Fps (18) and the mitochondrial protein septin (19), however the functional significance of these interactions remains unclear. The distribution of CRMP-5 in the growth cones suggests its potential role in regulating growth cone development and neurite outgrowth (15). However, the detailed mechanisms of CRMP-5 interaction with actin remain to be fully elucidated.

The current study aimed to determine whether CRMP-5 would interact with the actin cytoskeleton network to 
dynamically regulate the distribution and remodeling of cytoskeleton, thus to mediate growth cone development and neurite outgrowth.

\section{Materials and methods}

Animals. The experiments were conducted on 1-day-old pups of Sprague-Dawley rats $(\mathrm{n}=8-10)$. Rats were purchased from the Institute of Laboratory Animal Science of Jinan University (Jinan, China) and were sacrificed immediately. The rats were anesthetized with intraperitoneal injection of ketamine (30 mg/kg; Maijin Biotechnology, Hubei, China). Normally, the rats were housed in a temperature-controlled $\left(20-22^{\circ} \mathrm{C}\right)$ room with a $12 \mathrm{~h}$ light/dark cycle, and were provided with free access to food and water. All animal procedures were performed in strict accordance with the recommendations of the Guide for the Care and Use of Laboratory Animals from the National Institutes of Health (20). The protocol was approved by the Jinan University Institutional Animal Care and Use Committee (approval no. SCXK20110029; Guangzhou, China). All efforts were made to minimize the suffering and number of animals used.

Cell culture and transfection. Hippocampi were dissected from the postnatal rat pups (days 0-1), and dissociated hippocampal neurons were obtained using $0.125 \%$ trypsin (Gibco; Thermo Fisher Scientific Inc., Waltham, MA, USA), which were plated at a density of $1 \times 10^{4}$ cells $/ \mathrm{cm}^{2}$ onto poly-D-lysine (PDL)-coated glass coverslips. Cultures were maintained in Neurobasal-A (Gibco; Thermo Fisher Scientific Inc.) medium containing 2\% B27 (Gibco; Thermo Fisher Scientific Inc.) and $0.5 \mathrm{mM}$ glutamine (Gibco; Thermo Fisher Scientific Inc.) supplement at $37^{\circ} \mathrm{C}$ in a $5 \% \mathrm{CO}_{2}$ humidified incubator. Half of the culture media was replaced every 3 days. Calcium phosphate (Promega Corporation, Madison, WI, USA) transfections with different constructs were conducted on 6-7 days in vitro (DIV), and all experiments were performed on 7-8 DIV. Human embryonic kidney (HEK)293 cell (American Type Culture Collection, Manassas, VA, USA) culture was performed as described previously (21). To determine the expression of Flag-CRMP-5, the constructed pCMV-CRMP-5-Tag2 (pCMV-Tag2 vector was obtained from Addgene, Cambridge, MA, USA) was transfected into HEK293 cells using calcium phosphate. To verify the efficacy and specificity of siRNAs, co-transfection of 100 pmol siRNAs or NC together with $2 \mu \mathrm{g}$ of the pCMVCRMP-5-Tag2 plasmids into HEK293 cells was performed using calcium phosphate. Following transfection, cells were grown for $36 \mathrm{~h}$ prior to harvesting. Plasmids and siRNA fragments were transfected using the ProFection Mammalian Transfection system (Promega Corporation), performed according to the manufacturer's instructions.

Growth cone particle isolation. The methods were performed according to previous studies (22-24). Briefly, brains were dissected from the rats at 18 days of gestation and homogenized by a Teflon-glass homogenizer (Thomas Scientific, Swedesboro, NJ, USA) in $\sim 8$ volumes (w/v) of $0.32 \mathrm{M}$ sucrose (Sigma-Aldrich, St.Louis, MO, USA) containing $1 \mathrm{mM} \mathrm{MgCl}_{2}$ (Sigma-Aldrich) and $1 \mathrm{mM}$ Tes-NaOH (Sigma-Aldrich; $\mathrm{pH}$ 7.3), and the following protease inhibitors: $3 \mu \mathrm{M}$ aprotinin
(Calbiochem; EMD Millipore, Billerica, MA, USA), $20 \mathrm{mM}$ benzamidine, $1 \mathrm{mM}$ leupeptin, $1 \mathrm{mM}$ pepstatin A and $0.6 \mathrm{mM}$ phenylmethylsulfonyl fluoride (all from Sigma-Aldrich, St. Louis, MO, USA). The homogenate was centrifuged at $200 \mathrm{x} g$ for $15 \mathrm{~min}$. The low speed supernatant was loaded onto a discontinuous sucrose density gradient consisting of three layers: $0.75,1.0$ and $2.66 \mathrm{M}$; the gradients were spun to equilibrium at $150,000 \mathrm{x} \mathrm{g}$ for $200 \mathrm{~min}$ in a Beckman SW 40 Ti Rotor (Beckman Coulter, Inc., Brea, CA, USA). The isolated growth cone gradients were prepared as previously described (23). Neuronal samples subjected to immunocytochemistry were scanned by confocal microscopy. The fraction at the load/0.75 $\mathrm{M}$ interface (designated $\mathrm{A}$ as in the referred reference) contained the isolated growth cones or growth cone particles (GCPs). Then 'A' fraction samples were subjected to electron microscopy (H-7650, Hitachi, Tokyo, Japan).

Electron microscopic procedures were performed as preciously described (23). Briefly, aliquots were mixed slowly with increasing amounts of phosphate-buffered glutaraldehyde (1.5\%). After 10-15 min, the fixed material was pelleted by centrifugation at 2,000 $\mathrm{x}$ g for $5 \mathrm{~min}$ at room temperature into a conical embedding capsule (Electron Microscopy Sciences, Hatfield, PA, USA). After further glutaraldehyde fixation and washing with arsenate buffer, samples were treated with osmium tetroxide, block-stained with magnesium uranyl acetate and embedded in embedding mixture from the Low Viscosity Embedding Media Spurr's kit (all from Electron Microscopy Sciences). Thin sections were cut, stained and examined with the electron microscope.

Western blotting. Western blot analysis was performed as previously described (24). Briefly, lysates were separated using 8, 10 and $12 \%$ sodium dodecyl sulfate-polyacrylamide gel electrophoresis (Beyotime Institute of Biotechnology, Jiangsu, China) and were then electrophoretically transferred to a polyvinylidene difluoride membrane (Beyotime Institute of Biotechnology). Membranes were blocked in Tris-buffered saline (TBS; Beyotime Institute of Biotechnology) with 5\% milk and $0.05 \%$ Tween-20 and were then probed with the following primary antibodies at $4^{\circ} \mathrm{C}$ overnight: Rabbit anti-CRMP-5 (Santa Cruz Biotechnology, Inc., Santa Cruz, CA, USA, cat. no. sc-292382; $1: 1,000)$ and mouse anti- $\beta$-actin (cat. no. A5441; Sigma-Aldrich; 1:1,000). Subsequent to washing with TBS $+0.05 \%$ Tween; the membranes were incubated with horseradish peroxidase-conjugated goat anti-mouse (cat no. 115-001-008) or anti-rabbit secondary antibodies (cat no. 711-001-003; Jackson ImmunoResearch Laboratories, Inc., West Grove, PA, USA; 1:5,000) and were then visualized using enhanced chemiluminescence reagents (Beyotime Institute of Biotechnology).

Immunoprecipitation. Immunoprecipitation assays were performed as described previously $(21,25)$. For immunoprecipitation of hippocampal neurons, extracts were prepared by solubilization in $400 \mu \mathrm{l}$ of cell lysis buffer (Beyotime Institute of Biotechnology) for $10 \mathrm{~min}$ at $4^{\circ} \mathrm{C}$. Subsequent to brief sonication, the lysates were cleared by centrifugation at $15,000 \mathrm{x} \mathrm{g}$ for $10 \mathrm{~min}$ at $4^{\circ} \mathrm{C}$, the cell extract was then immunoprecipitated with $4 \mu \mathrm{g}$ of the antibodies against CRMP-5 $(1: 500)$ or $\beta$-actin $(1: 1,000)$, then the samples were incubated 
A

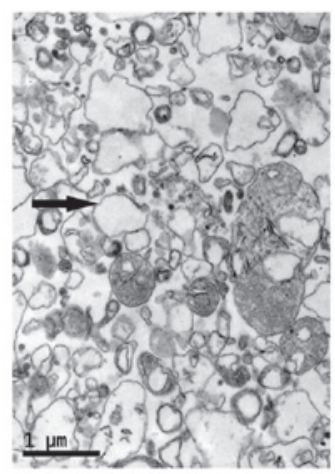

B

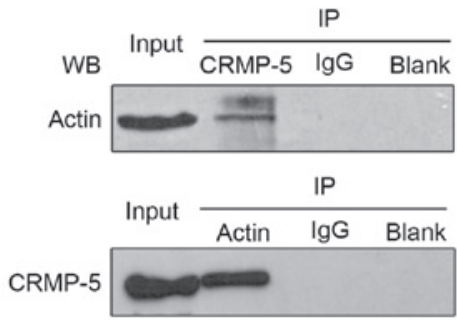

C

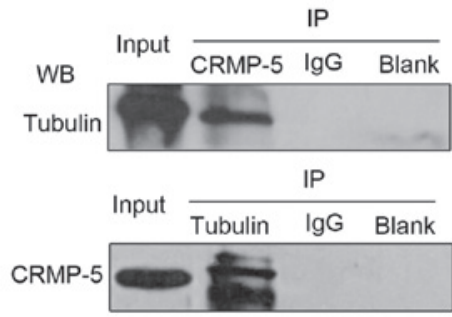

Figure 1. CRMP-5 interacts with the cytoskeleton. (A) Purified rat growth cone lysates were subjected to transmission electron microscopy. Growth cone lysates from rat brains were subjected to IP and WB analysis with (B) actin and CRMP-5 antibodies and (C) tubulin and CRMP-5 antibodies. CRMP-5, collapsin response mediator protein 5; IP, immunoprecipitation; WB, western blotting; blank, A/G group with no antibody.

with $60 \mu \mathrm{l}$ of protein $\mathrm{G}$ plus protein A-agarose (Calbiochem; EMD Millipore) for $16 \mathrm{~h}$ at $4^{\circ} \mathrm{C}$ by continuous inversion. Immunocomplexes were pelleted at $500 \mathrm{xg}$ for $5 \mathrm{~min}$ at $4^{\circ} \mathrm{C}$ and washed three times. The precipitated immunocomplexes were boiled in Laemmli buffer and assayed using western blot analysis with anti-CRMP-5 or mouse monoclonal anti- $\beta$-tubulin antibodies (Sigma-Aldrich; cat. no. T5201; dilution, 1:2,000).

Immunofluorescence. Hippocampal neurons were grown on coverslips (Thermo Fisher Scientific, Inc.) and were processed for immunofluorescence according to the standard protocol described previously (24). Cells were fixed with $4 \%$ (w/v) paraformaldehyde (Sigma-Aldrich) for $5 \mathrm{~min}$ at room temperature and permeabilized with $0.1 \%$ Triton $\mathrm{X}-100$ in phosphate-buffered saline (PBS) for $20 \mathrm{~min}$. The cells were blocked in 3\% normal donkey serum (Jackson Immunoresearch Laboratories, Inc.) in TBS $+0.1 \%$ Triton X-100 for $1 \mathrm{~h}$ at room temperature and incubated with the rabbit anti-CRMP-5 and mouse anti-actin antibodies at $4^{\circ} \mathrm{C}$ overnight. The cells were washed 3 times for 10 min with PBS $+0.1 \%$ Tween-20, and were incubated with the monoclonal donkey anti-rabbit $\mathrm{IgG}$ Dylight 549 (Jackson ImmunoResearch Laboratories, Inc.; cat. no. 711-516-152; 1:1,000) or monoclonal donkey anti-mouse IgG Dylight 488 (Jackson ImmunoResearch Laboratories, Inc.; cat. no. 715-485-150; 1:1,000) for $2 \mathrm{~h}$ at room temperature. Subsequent to three washes in lysis buffer, cells were mounted on glass slides with Fluoro Gel II containing 4',6-diamidino-2-phenylindole (Electron Microscopy Sciences, Hatfield, PA, USA). For co-localization analysis, confocal images were analyzed using ImageJ software (version 1.48, National Institutes of Health, Bethesda, MA, USA) with Just Another Colocalization Plugin (JACoP, National Institutes of Health) (26). The random or codependent nature of colocalizations were tested using Cytofluorogram and intensity correlation analysis. All the co-localization calculations were performed on $>10$ cells from at least three independent experiments. Microscopy and image analysis were conducted using the same optical slice thickness for every channel using a confocal microscope (LSM 710; Carl Zeiss AG, Oberkochen, Germany).

RNA interference. A validated CRMP-5 short interfering RNA (siRNA) (siCRMP-5) fragment and NC (scrambled sequence, negative control) were synthesized by Shanghai GenePharma Co., Ltd. (Shanghai, China) and validated previously $(15,17,24)$. Hippocampal neurons were transfected with siRNA using a calcium phosphate protocol (27). To transfect neurons in 24-well tissue culture plates, 100 pmol siRNA was combined with $37 \mu 12 \mathrm{M} \mathrm{CaCl}_{2}$ solution in sterile, deionized water to a final volume of $300 \mu \mathrm{l}$ and then mixed well with $300 \mu 12 X$ 4-(2-hydroxyethyl)-1-piperazineethanesulfonic acid-buffered saline. The mixtures were vortexed and incubated at $25^{\circ} \mathrm{C}$ for approximately $4 \mathrm{~min}$. In each well, $30 \mu \mathrm{l}$ mixture was added drop-wise to the cells and allowed to incubate for an additional $25 \mathrm{~min}$. The green fluorescent protein expression plasmid (Addgene) was co-transfected with the siRNAs to mark the transfected cells.

Statistical analysis. Data are presented as the mean \pm standard error. Significant differences were assessed with one-way analysis of variance followed by the Bonferroni or Tamhane post hoc tests. $\mathrm{P}<0.05$ was considered to indicate a statistically significant difference.

\section{Results}

CRMP-5 interacts with cytoskeleton. In order to separate GCPs from the soma, sucrose gradient centrifugation was used. GCPs were then subjected to transmission electron microscopy. As presented in Fig. 1A, GCPs existed as vesicular structures (indicated by arrows), with a uniform size $(\sim 1 \mu \mathrm{m})$ and loose distribution, which was consistent with previously reported results (22). Subsequently, co-immunoprecipitation was conducted in order to determine whether CRMP-5 would interact with the actin and tubulin cytoskeletons, rather than with tubulin alone as previously reported (24). Using the CRMP-5 antibody, the actin immune-signal in the CRMP-5-precipitated sediment from GCP lysates was detected, with no actin immune-signal in the IgG and blank control groups (Fig. 1B, upper panel). Conversely, using the actin antibody, a CRMP-5 signal was detected in the actin-precipitated sediment (Fig. 1B, lower panel). Furthermore, CRMP-5 was indicated to interact with tubulin using the CRMP-5 and tubulin antibodies (Fig. 1C). These data suggest that CRMP-5 interacts with the actin and tubulin cytoskeleton systems. 
A

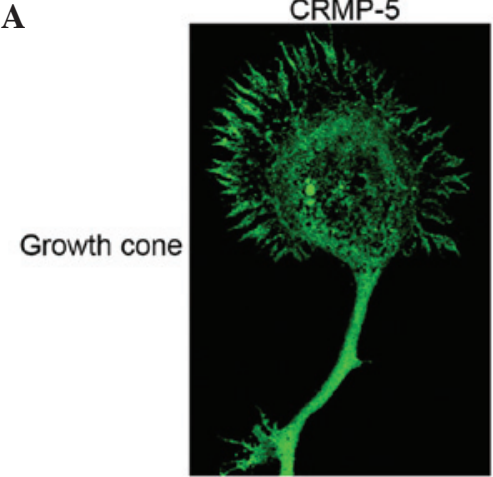

B

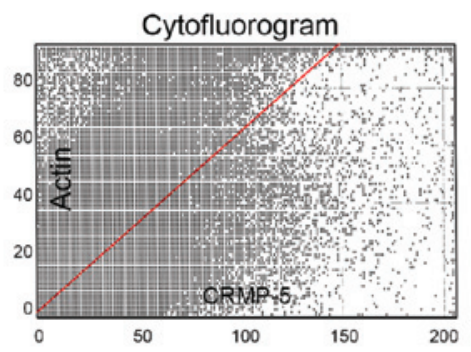

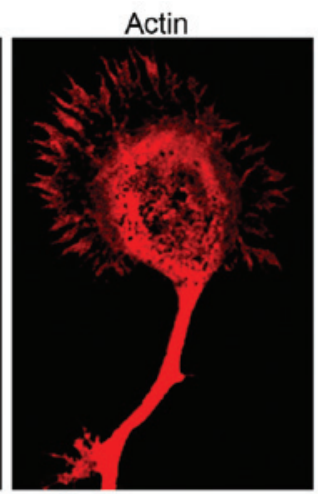

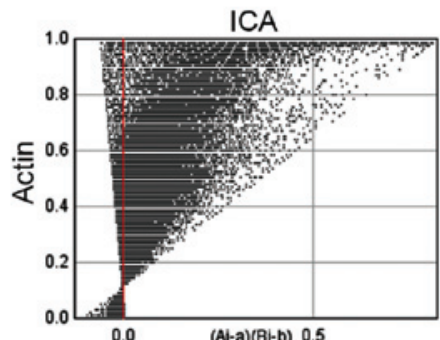

Merge
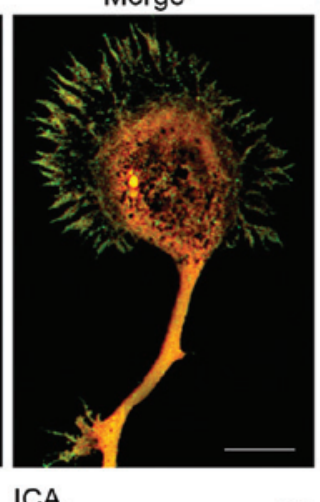

Colocalized area (white)

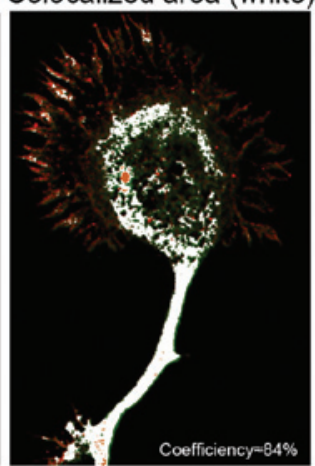

ICA

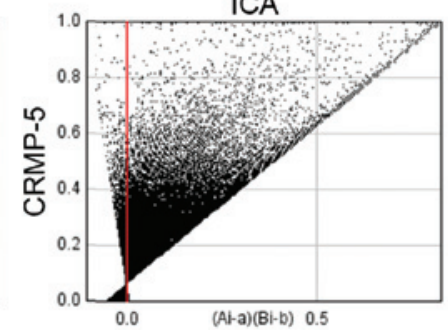

Figure 2. CRMP-5 colocalizes with actin in growth cones of hippocampal neurons. (A) Anti-actin and anti-CRMP-5 antibodies were used to detect endogenous actin and CRMP-5 proteins, respectively, in hippocampal neurons cultured for $72 \mathrm{~h}$. The merged images present the colocalization (yellow) and colocalized area (white) of CRMP-5 (green) and actin (red). Scale bar, $10 \mu \mathrm{m}$. (B) The cytofluorogram and ICA for the colocalization analysis of actin and CRMP-5 in (A) is presented. CRMP-5, collapsin response mediator protein 5; ICA, intensity correlation analysis.

A

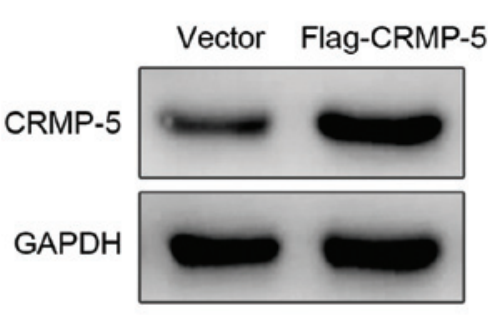

B

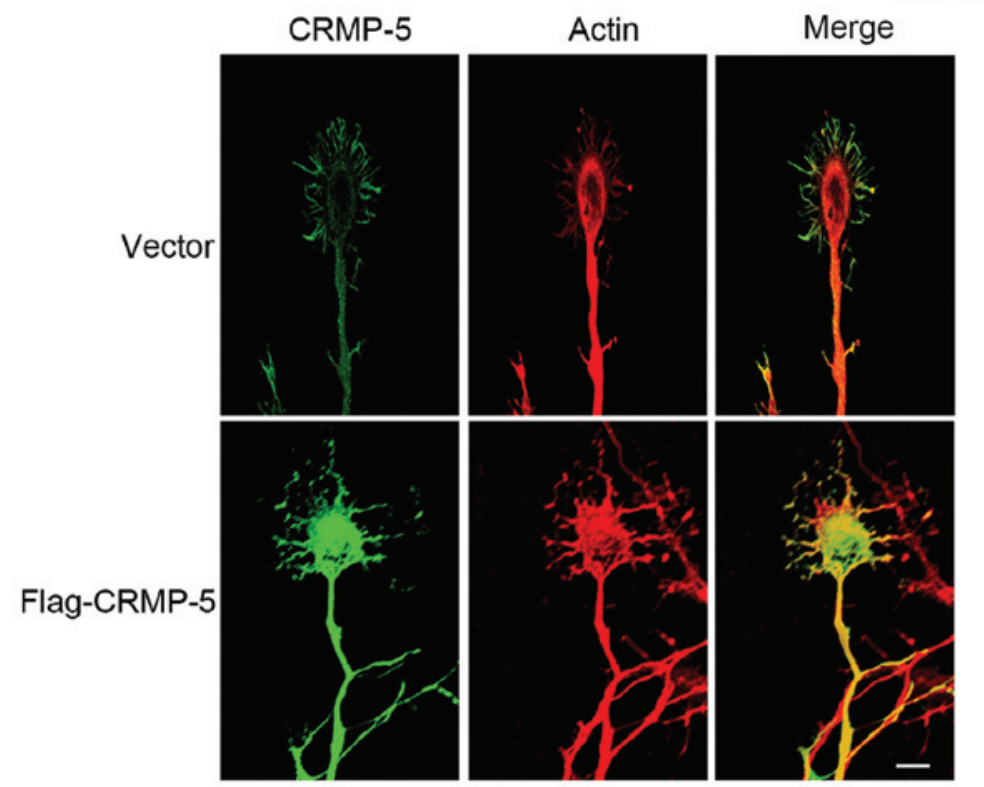

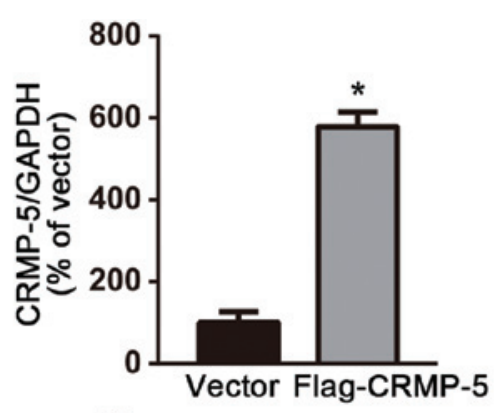

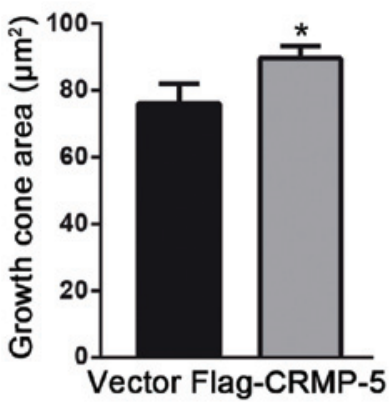

Figure 3. CRMP-5 is sufficient for actin dynamics and growth cone development. (A) HEK293 cells were transfected with the FLAG-CRMP-5 expression plasmid or the control vector. CRMP-5 expression was detected by immunoblotting using an anti-FLAG antibody. The GAPDH antibody was used as a loading control. (B) Representative images of growth cones of neurons transfected with the FLAG-CRMP-5 expression plasmid or the control vector are presented. The growth cone area of transfected cells was measured and plotted. Comparisons were made using one-way analysis of variance. Data are presented as the mean \pm standard error from three independent experiments, "P<0.05. Scale bar, $10 \mu \mathrm{m}$. CRMP-5, collapsin response mediator protein 5; GAPDH, glyceraldehyde 3-phosphate dehydrogenase. 
A
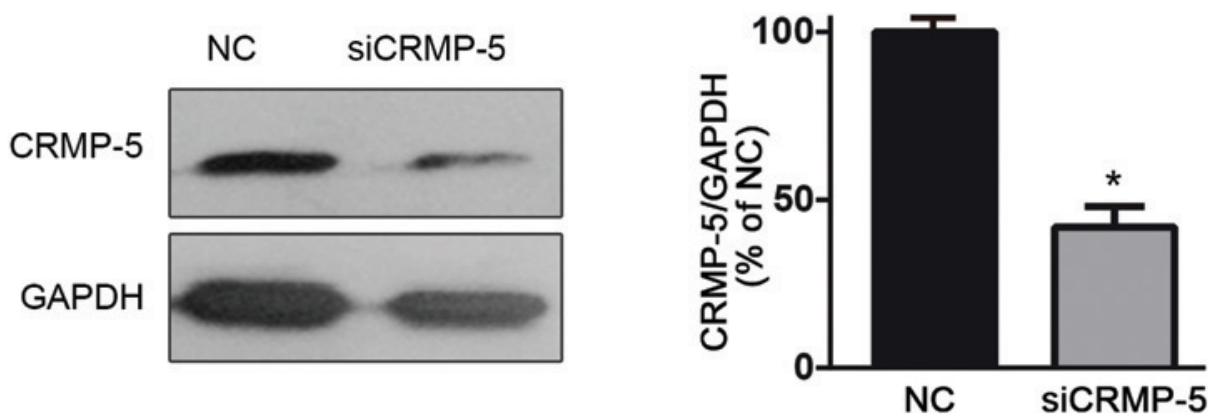

B

GFP

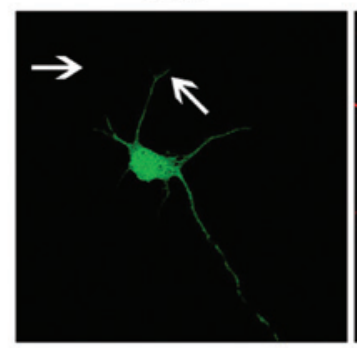

Actin

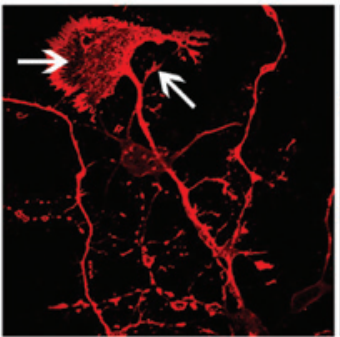

Merge

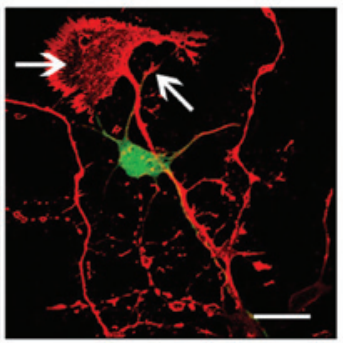

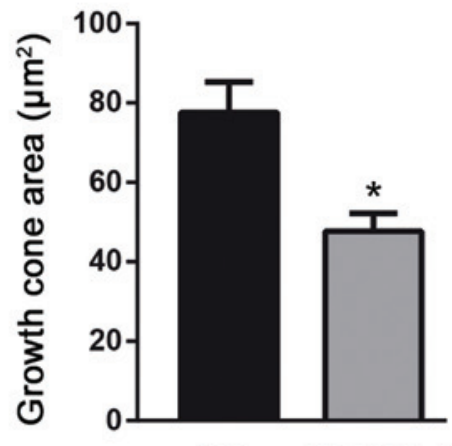

NC SiCRMP-5

Figure 4. CRMP-5 is necessary for actin dynamics and growth cone development. (A) HEK293 cells were co-transfected with scrambled siRNA (NC) or siCRMP-5 together with the FLAG-CRMP-5 expression plasmid. Lysates were probed with an anti-CRMP-5 antibody. The GAPDH antibody was used as a loading control. Statistical data of siRNA efficiency is presented as the mean \pm standard error for three independent experiments. ${ }^{*}<0.05$. (B) Hippocampal neurons cultured for $48 \mathrm{~h}$ were co-transfected with scrambled siRNA (NC) or CRMP-5-siRNA together with a GFP-encoding plasmid; after 24 h, the neurons were fixed and subjected to immunocytochemistry. Typical images of the CRMP-5 and actin immunostaining are presented. The growth cone areas of transfected cells were determined and plotted. * $\mathrm{P}<0.05$ ( $\mathrm{n}=30-40$ cells from 3 independent experiments). Error bars indicate standard error of the mean. Scale bar, $10 \mu \mathrm{m}$. CRMP-5, collapsin response mediator protein 5; siRNA, short interfering RNA; NC, negative control; GAPDH, glyceraldehyde 3-phosphate dehydrogenase; GFP, green fluorescent protein.

A
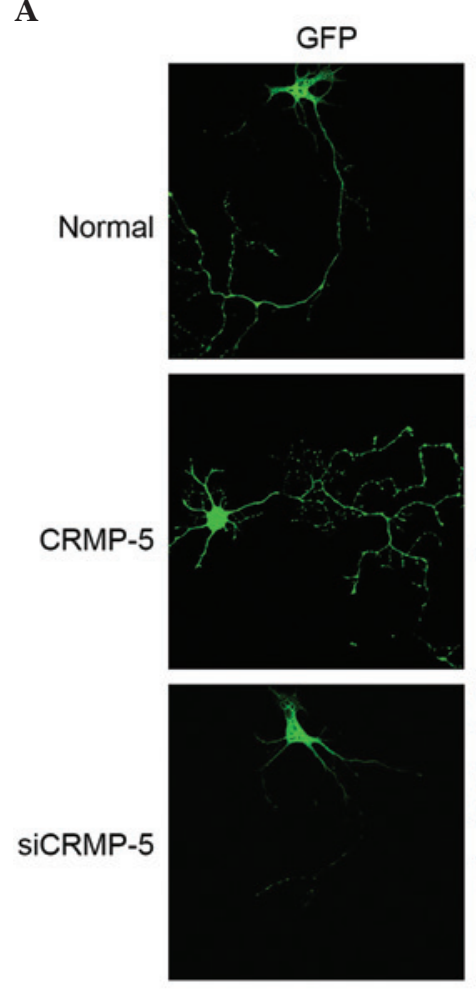

Tubulin
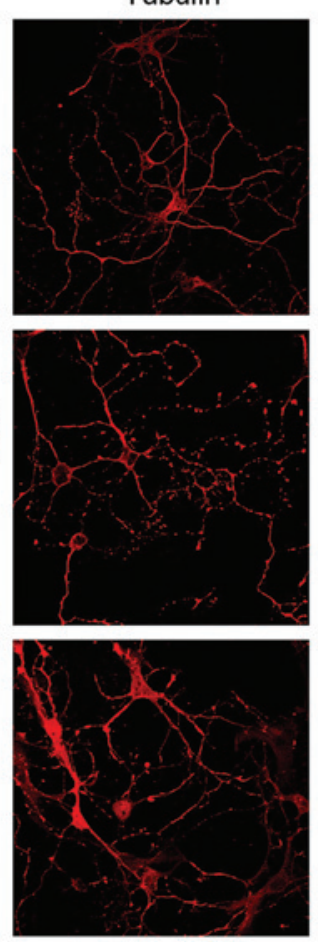
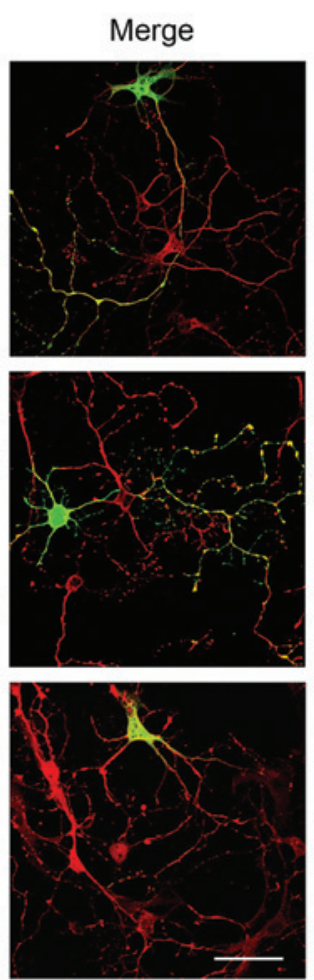

B

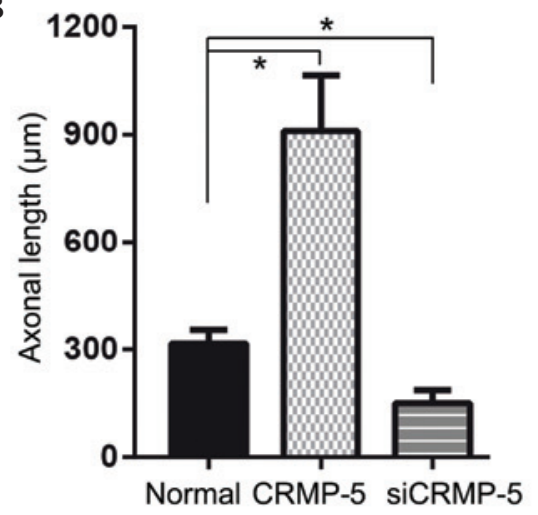

C

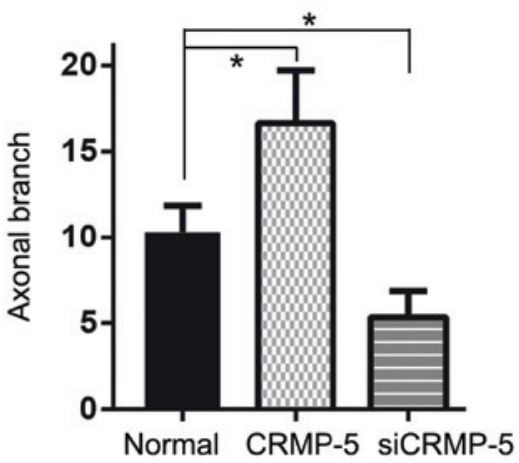

Figure 5. CRMP-5 regulates neurite outgrowth. (A) Representative images of neurons transfected with the CRMP-5 overexpression plasmid or siRNA fragments were stained with GFP and tubulin to indicate neurite outgrowth. Statistical data of (B) neurite length and (C) neurite branch number are presented as the mean \pm standard error; $n=5,{ }^{*} \mathrm{P}<0.05$. CRMP-5, collapsin response mediator protein 5; siRNA, short interfering RNA; GFP, green fluorescent protein. 
CRMP-5 colocalizes with actin in growthcones of hippocampal neurons. Furthermore, the distribution of actin and CRMP-5 in growth cones of developing hippocampal neurons was investigated. Neurons were cultured at a density of $1 \times 10^{4}$ cells $/ \mathrm{cm}^{2}$ on a PDL-coated coverslip. At 3 DIV, neurons were subjected to immunofluorescence with CRMP-5 and actin antibodies. As presented in Fig. 2, actin was predominantly distributed in the P-domain and T-zone, with fewer signals in the C-domain. Lamellipodia in the P-domain exhibited a clear mesh and bundled filaments. CRMP-5 was distributed predominantly in the C-domain and T-zone, however also exhibited clear signals in the lamellipodia and filopodia of the P-domain. CRMP-5 and actin were observed to exhibit colocalization in the actin filaments in the P-domain and in the T-zone, with a colocalization co-efficiency of $84 \%$ (Fig. 2, right panel). Using analysis with ImageJ plus JACoP (26), a clear cytofluorogram and ICA score between actin and CRMP-5 was obtained, which additionally indicted colocalization of actin and CRMP-5. These data suggest that CRMP-5 colocalizes with actin in the growth cones of developing neurons.

CRMP-5 regulates actin dynamics and growth cone development. In order to determine the effect of CRMP-5 on actin dynamics and growth cone development, a CRMP-5 expression plasmid (pCMV-CRMP-5-Tag2) was constructed, which had been previously reported (26). As presented in Fig. 3A, the CRMP-5 plasmid was successfully expressed in HEK293 cells by the detection of western blot with the Flag antibody. The plasmid was then transfected into hippocampal neurons. As presented in Fig. 3B, growth cones transfected with CRMP-5 were fan-shaped, with actin predominantly distributed in the P-domain with long actin filaments and increased branches. Overexpression of CRMP-5 promoted growth cone development, with an enlarged growth cone area (Fig. 3B, right panel). The CRMP-5 siRNA experiment was then conducted, where the siRNA efficiency was determined previously (24) and the results are presented in Fig. 4A. On CRMP-5 silencing, the actin filaments were observed to become fragile, and exhibited collapsed growth cones, compared with those in non-transfected neurons (Fig. 4B). These data suggest that CRMP-5 regulates actin dynamics and growth cone development.

CRMP-5 regulates neurite outgrowth. It was then investigated whether CRMP-5 would regulate neurite outgrowth. Hippocampal neurons were transfected with the CRMP-5 overexpression plasmid or with CRMP-5 siRNA fragments. Subsequent to transfection for 5 days, the neurons were subjected to immunocytochemistry. As presented in Fig. 5, the CRMP-5-overexpressing neurons were observed to have a longer neurite length and an increased number of neurite branches than that of CRMP-5-silenced neurons, which possessed short neurite length and few branches (Fig. 5B and C). These data suggest that CRMP-5 regulates neurite outgrowth via modulating actin dynamics and growth cone development.

\section{Discussion}

CRMPs are critical for growth cone development, axonal guidance and neuronal polarity. CRMP proteins 1-5 have been implicated to associated with tubulin $(17,28)$. CRMP-5, an isoform distinct from the other four CRMPs, was recently reported to localize in the filopodia of growth cones (15), leaving the role of CRMP-5-associated proteins in regulating growth cones to be further elucidated. Thus, in the current study, it was identified that CRMP-5 interacted with the actin cytoskeleton, colocalized with actin in growth cones, regulated actin dynamics and modulated growth cone development and neurite outgrowth.

Neurite outgrowth relies on the interaction between the actin and microtubule cytoskeleton (29). Actin and microtubules within the growth cone are not independent, however are associated, sensing each other's movement (29). Heidemann et al (30) identified that coordinated cytoskeletal movement was important in axon guidance. Numerous signaling transduction pathways and proteins have been reported to be involved in this process. These proteins include Rho family proteins (31-33), plus-end tracking proteins $(34,35)$, spectraplakins $(36,37)$ and microtubule-associated proteins (38), particularly tau protein (39-41). In addition, CRMPs may be the structural mediator of actin and microtubules, CRMP-2 is able to interact with tubulin promoting its assembly $(28,42)$. CRMP-4 interacts with actin and promotes neurite growth $(43,44)$. Previous studies have identified that CRMP-5 interacts with tubulin in the growth cones (24). It has additionally been identified that CRMP-2 and CRMP-4 interact to coordinate cytoskeletal dynamics, regulating growth cone development and axon elongation (45). Whether additional novel proteins or members of the CRMP family, (e.g. CRMP-1 and CRMP-3) are involved in this process remains unclear, thus requires further investigation.

CRMP-5 is highly distributed in fetal and neonatal rat brains and is markedly reduced in adult brains (13). In hippocampal neurons, the spatiotemporal distribution of CRMP-5 varies at different developmental stages; CRMP-5 is present in growth cones at stage 3 when neuronal polarity is developed, and reduces to a low level when neurons are polarized. Thus, CRMP-5 contributes to the dynamic regulation of neuronal polarity (17). The data of the current study are consistent with previously reported data (15), in that CRMP-5 in the growth cone is necessary and sufficient for growth cone development. The results of the current study additionally suggest that CRMP-5 functions through its interaction with the cytoskeleton. CRMP-5 has been reported to inhibit dendritic growth in hippocampal neurons (17). However, in cerebellar Purkinje cells, CRMP-5 has been previously reported to promote dendritic development and synaptic plasticity in studies using CRMP-5 ${ }^{-/}$mice, where aberrant dendrite morphology was observed $(16,17)$. The different functions of CRMP-5 from these studies suggest that CRMP-5 acts to be cell-type specific and this may be due to its efferent distribution or its different interacting proteins. Taken together, the current study has provided novel evidence for CRMP-5-regulated growth cone development and neurite outgrowth.

\section{Acknowledgements}

The present study was supported by the National Natural Science Foundation of China (grant nos. 31170941 and 31300885), the Natural Science Foundation of Guangdong 
Province, China (grant nos. S2013010014191, 2014A030310024 and 2014A030313357), Medical Scientific Research Foundation of Guangdong Province, China (grant nos. A2014382 and A2015479) and the Fundamental Research Funds for the Central Universities (grant no. 21615477).

\section{References}

1. Huber AB, Kolodkin AL, Ginty DD and Cloutier JF: Signaling at the growth cone: Ligand-receptor complexes and the control of axon growth and guidance. Annu Rev Neurosci 26: 509-563, 2003

2. Tessier-Lavigne M and Goodman CS: The molecular biology of axon guidance. Science 274: 1123-1133, 1996.

3. Rolls MM and Jegla TJ: Neuronal polarity: an evolutionary perspective. J Exp Biol 218: 572-580, 2015.

4. Nieto MA: Molecular biology of axon guidance. Neuron 17: 1039-1048, 1996.

5. Zou Y: Does planar cell polarity signaling steer growth cones? Curr Top Dev Biol 101: 141-160, 2012.

6. Pak CW, Flynn KC and Bamburg JR: Actin-binding proteins take the reins in growth cones. Nat Rev Neurosci 9: 136-147, 2008.

7. Schaefer AW, Kabir N and Forscher P: Filopodia and actin arcs guide the assembly and transport of two populations of microtubules with unique dynamic parameters in neuronal growth cones. J Cell Biol 158: 139-152, 2002.

8. Buck KB and Zheng JQ: Growth cone turning induced by direct local modification of microtubule dynamics. J Neurosci 22 9358-9367, 2002.

9. Minturn JE, Fryer HJ, Geschwind DH and Hockfield S: TOAD-64, a gene expressed early in neuronal differentiation in the rat, is related to unc-33, a C. elegans gene involved in axon outgrowth. J Neurosci 15: 6757-6766, 1995.

10. Fukada M, Watakabe I, Yuasa-Kawada J, Kawachi H, Kuroiwa A, Matsuda Y and Noda M: Molecular characterization of CRMP5, a novel member of the collapsin response mediator protein family. J Biol Chem 275: 37957-37965, 2000.

11. Yuasa-Kawada J, Suzuki R, Kano F, Ohkawara T, Murata M and Noda M: Axonal morphogenesis controlled by antagonistic roles of two CRMP subtypes in microtubule organization. Eur J Neurosci 17: 2329-2343, 2003

12. Goshima Y, Nakamura F, Strittmatter P and Strittmatter SM: Collapsin-induced growth cone collapse mediated by an intracellular protein related to UNC-33. Nature 376: 509-514, 1995.

13. Inatome R, Tsujimura T, Hitomi T, Mitsui N, Hermann $P$, Kuroda S, Yamamura H and Yanagi S: Identification of CRAM, a novel unc-33 gene family protein that associates with CRMP3 and protein-tyrosine kinase(s) in the developing rat brain. J Biol Chem 275: 27291-27302, 2000.

14. Ricard D, Rogemond V, Charrier E, Aguera M, Bagnard D, Belin MF, Thomasset $\mathrm{N}$ and Honnorat J: Isolation and expression pattern of human Unc-33-like phosphoprotein 6/collapsin response mediator protein 5 (Ulip6/CRMP5): Coexistence with Ulip2/CRMP2 in Sema3a- sensitive oligodendrocytes. J Neurosci 21: 7203-7214, 2001.

15. Hotta A, Inatome R, Yuasa-Kawada J, Qin Q, Yamamura H and Yanagi S: Critical role of collapsin response mediator protein-associated molecule CRAM for filopodia and growth cone development in neurons. Molecular Biology of the Cell 16: 32-39, 2005.

16. Yamashita N, Mosinger B, Roy A, Miyazaki M, Ugajin K, Nakamura F, Sasaki Y, Yamaguchi K, Kolattukudy P and Goshima Y: CRMP5 (collapsin response mediator protein 5) regulates dendritic development and synaptic plasticity in the cerebellar Purkinje cells. J Neurosci 31: 1773-1779, 2011.

17. Brot S, Rogemond V, Perrot V, Chounlamountri N, Auger C, Honnorat J and Moradi-Améli M: CRMP5 interacts with tubulin to inhibit neurite outgrowth, thereby modulating the function of CRMP2. J Neurosci 30: 10639-10654, 2010.

18. Mitsui N, Inatome R, Takahashi S, Goshima Y, Yamamura H and Yanagi S: Involvement of Fes/Fps tyrosine kinase in semaphorin3A signaling. EMBO J 21: 3274-3285, 2002.

19. National Research Council: Guide for the Care and Use of Laboratory Animals. National Academy Press: Washington DC; 1996: 125.

20. Takahashi S, Inatome R, Yamamura $H$ and Yanagi S: Isolation and expression of a novel mitochondrial septin that interacts with CRMP/CRAM in the developing neurones. Genes Cells 8: 81-93, 2003.
21. Zhang J, Fan J, Tian Q, Song Z, Zhang JF and Chen Y: Characterization of two distinct modes of endophilin in clathrin-mediated endocytosis. Cell Signal 24: 2043-2050, 2012.

22. Pfenninger KH, Ellis L, Johnson MP, Friedman LB and Somlo S: Nerve growth cones isolated from fetal rat brain: Subcellular fractionation and characterization. Cell 35: 573-584, 1983 .

23. Lohse K, Helmke SM, Wood MR, Quiroga S, de la Houssaye BA, Miller VE, Negre-Aminou P and Pfenninger KH: Axonal origin and purity of growth cones isolated from fetal rat brain. Brain Res Dev Brain Res 96: 83-96, 1996.

24. Xiao L, Liu W, Li J, Xie Y, He M, Fu J, Jin W and Shao C: Irradiated U937 cells trigger inflammatory bystander responses in human umbilical vein endothelial cells through the p38 pathway. Radiat Res 182: 111-121, 2014.

25. Tian Q, Zhang JF, Fan J, Song Z and Chen Y: Endophilin isoforms have distinct characteristics in interactions with N-type Ca2+ channels and dynamin I. Neurosci Bull 28: 483-492, 2012.

26. Bolte S and Cordelières FP: A guided tour into subcellular colocalization analysis in light microscopy. J Microsc 224: 213-232, 2006

27. Tan M, Ma S, Huang Q, Hu K, Song B and Li M: GSK- $3 \alpha / \beta$-mediated phosphorylation of CRMP-2 regulates activity-dependent dendritic growth. J Neurochem 125: 685-697, 2013.

28. Fukata Y, Itoh TJ, Kimura T, Ménager C, Nishimura T, Shiromizu T, Watanabe H, Inagaki N, Iwamatsu A, Hotani $\mathrm{H}$ and Kaibuchi K: CRMP-2 binds to tubulin heterodimers to promote microtubule assembly. Nat Cell Biol 4: 583-591, 2002.

29. Dent EW, Gupton SL and Gertler FB: The growth cone cytoskeleton in axon outgrowth and guidance. Cold Spring Harb Perspect Biol 3: a001800, 2011.

30. Heidemann SR, Landers JM and Hamborg MA: Polarity orientation of axonal microtubules. J Cell Biol 91: 661-665, 1981.

31. Ren XD, Kiosses WB and Schwartz MA: Regulation of the small GTP-binding protein Rho by cell adhesion and the cytoskeleton. EMBO J 18: 578-585, 1999.

32. Waterman-Storer CM, Worthylake RA, Liu BP, Burridge K and Salmon ED: Microtubule growth activates Racl to promote lamellipodial protrusion in fibroblasts. Nat Cell Biol 1: 45-50, 1999.

33. Watabe-Uchida M, Govek EE and Van Aelst L: Regulators of Rho GTPases in neuronal development. J Neurosci 26: 10633-10635, 2006.

34. Watanabe T, Noritake J and Kaibuchi K: Roles of IQGAP1 in cell polarization and migration. Novartis Found Symp 269: 92-101; discussion 101-105, 223-230, 2005.

35. Komarova Y, Lansbergen G, Galjart N, Grosveld F, Borisy GG and Akhmanova A: EB1 and EB3 control CLIP dissociation from the ends of growing microtubules. Mol Biol Cell 16: 5334-5345, 2005.

36. Suozzi KC, Wu X and Fuchs E: Spectraplakins: Master orchestrators of cytoskeletal dynamics. J Cell Biol 197: 465-475, 2012

37. Kodama A, Lechler T and Fuchs E: Coordinating cytoskeletal tracks to polarize cellular movements. J Cell Biol 167: 203-207, 2004.

38. Cueille N, Blanc CT, Popa-Nita S, Kasas S, Catsicas S, Dietler G and Riederer BM: Characterization of MAP1B heavy chain interaction with actin. Brain Res Bull 71: 610-618, 2007.

39. He HJ, Wang XS, Pan R, Wang DL, Liu MN and He RQ: The proline-rich domain of tau plays a role in interactions with actin. BMC Cell Biol 10: 81, 2009.

40. Correas I, Padilla R and Avila J: The tubulin-binding sequence of brain microtubule-associated proteins, tau and MAP-2, is also involved in actin binding. Biochem J 269: 61-64, 1990.

41. Henríquez JP, Cross D, Vial C and Maccioni RB: Subpopulations of tau interact with microtubules and actin filaments in various cell types. Cell Biochem Funct 13: 239-250, 1995.

42. Arimura N, Menager C, Fukata Y and Kaibuchi K: Role of CRMP-2 in neuronal polarity. J Neurobiol 58: 34-47, 2004.

43. Rosslenbroich V, Dai L, Baader SL, Noegel AA, Gieselmann V and Kappler J: Collapsin response mediator protein-4 regulates F-actin bundling. Exp Cell Res 310: 434-444, 2005.

44. Quinn CC, Chen E, Kinjo TG, Kelly G, Bell AW, Elliott RC, McPherson PS and Hockfield S: TUC-4b, a novel TUC family variant, regulates neurite outgrowth and associates with vesicles in the growth cone. J Neurosci 23: 2815-2823, 2003.

45. Tan M, Cha C, Ye Y, Zhang J, Li S, Wu F, Gong S and Guo G: CRMP4 and CRMP2 interact to coordinate cytoskeleton dynamics, regulating growth cone development and axon elongation. Neural Plast 2015: 947423, 2015. 